\title{
Pretreatment of BMSCs with TZD solution decreases the proliferation rate of MCF-7 cells by reducing FGF4 protein expression
}

\author{
BOON-YIN KHOO ${ }^{1,2}$, KALPANAH NADARAJAN ${ }^{1}$, SIANG-YIAN SHIM ${ }^{1}$, NOORIZAN MISWAN ${ }^{1}$, \\ CHUAN-BING ZANG ${ }^{2}$, KURT POSSINGER ${ }^{2}$ and ELENA ELSTNER ${ }^{2}$
}

\author{
${ }^{1}$ Institute for Research in Molecular Medicine (INFORMM), Universiti Sains Malaysia, Penang 11800, Malaysia; \\ ${ }^{2}$ Division of Oncology and Haematology, Charité Campus Mitte, Humboldt University of Berlin, D-10099 Berlin, Germany
}

Received May 19, 2015; Accepted February 4, 2016

DOI: $10.3892 / \mathrm{mmr} .2016 .4959$

\begin{abstract}
The present study aimed to investigate the effects of bone marrow-derived mesenchymal stem cells (BMSCs) that had been pretreated with pioglitazone and/or rosiglitazone on the growth and proliferation rate of MCF-7 cells. The adhesive interaction between the BMSCs and the MCF-7 cancer cells revealed that the pretreatment of BMSCs with a combination of two types of thiazolidinedione drug reduced the growth and proliferation rate of the MCF-7 cells. The proliferation rate of the MCF-7 cells could also be reduced by the non-adhesive interaction of the cancer cells with BMSCs pretreated with pioglitazone and/or rosiglitazone. The growth and proliferation rate reduction effects on the MCF-7 cells may be attributed to the reduction in the protein level of fibroblast growth factor 4 (FGF4) in the conditioned medium of the pretreated BMSCs. The evidence that the low protein level of FGF4 in the conditioned medium of the pretreated BMSCs perturbed the proliferation rate of the MCF-7 cells by reducing the levels of Ki-67 and proliferating cell nuclear antigen transcripts in the cancer cells was also demonstrated in the present study using a FGF4-neutralizing antibody. All the above findings demonstrate that future studies on the correlation between FGF4 and pretreated BMSCs would be beneficial.
\end{abstract}

\section{Introduction}

Mesenchymal stem cells (MSCs) are progenitor cells that have been described to localize within breast carcinomas, where stem cells integrate into tumor-associated stromal tissues and

Correspondence to: Dr Boon-Yin Khoo, Institute for Research in Molecular Medicine (INFORMM), Universiti Sains Malaysia, Penang 11800, Malaysia

E-mail: boonyin@usm.my; kboonyin@yahoo.com

Key words: bone marrow-derived mesenchymal stem cells, TZD solution, MCF-7, stem- and -cancer cell interaction, cell growth, proliferation rate, FGF4 promote breast cancer invasion and metastasis $(1,2)$. The MSCs isolated from bone marrow have been demonstrated to greatly increase the metastatic potency of weakly metastatic human breast carcinoma cells (3). This phenomenon was observed in MCF-7 cells, where an increase in cancer cell proliferation was observed when the cancer cells were co-cultured on bone marrow-derived MSC (BMSC) feeder layers. Thus, this co-culture of BMSCs and cancer cells may be used as a model to identify a potential method for reducing the propagation characteristics (growth and proliferation rate) of cancer cells.

The present study aimed to investigate the growth reduction effects of BMSCs that had been pretreated with pioglitazone and/or rosiglitazone on the growth and proliferation rate of breast cancer cells. Pioglitazone and rosiglitazone are prescription drugs of the thiazolidinedione (TZD) class that are commonly used for the treatment of type II diabetes mellitus. Although pioglitazone and rosiglitazone are currently widely used clinically, neither pioglitazone nor rosiglitazone have any role in the treatment of human breast cancer. Indeed, the use of these drugs to treat cancer cells is not necessarily a promising strategy in breast cancer therapy. For example, although pioglitazone and rosiglitazone have been demonstrated to reduce breast cancer cell proliferation and invasion $(4,5)$, these drugs, however, also demonstrate certain disadvantages, including cellular lipid accumulation, as determined from an in vitro assay, heart failure and bone destruction in female patients. Therefore, it would be beneficial to administer pioglitazone and rosiglitazone indirectly to breast cancer patients, for example, via the interaction of stem and cancer cells. Through this process, the modified and viable pretreated stem cells would be subsequently administered to patients, and the cells would allowed to interact with cancer cells in the body of the patients.

In the present study, the effect of soluble growth factors in the conditioned medium of the pretreated BMSCs on the proliferation rate of MCF-7 cells was investigated using a fibroblast growth factor 4 (FGF4) neutralizing antibody. It was hypothesized that the pretreated stem cells would reduce cancer cell growth (colony size) and the proliferation rate (colony number) in vitro (Fig. 1). This phenomenon may be attributed to the reduction of specific soluble growth factors in the pretreated 
BMSCs; therefore, studying the expression pattern of growth and inflammatory response-associated molecules, including FGF4, chemokine (C-C motif) ligand-5 (CCL5; also termed RANTES) and interleukin-6 (IL-6), may provide insights into the regulation of stem cells in carcinogenesis. The results of the present study may also provide valuable insights into the usefulness of pioglitazone- and/or rosiglitazone-pretreated BMSCs, which may expand the benefits of using pretreated BMSCs in future medical studies. The pioglitazone- and/or rosiglitazone-pretreated BMSCs may also have a potential application in stem cell-mediated therapy for human breast cancer, as well as for other malignancies.

\section{Materials and methods}

Culture of the BMSCs and MCF-7 cell lines. The BMSC cell line was purchased from AseaCyte Sdn Bhd (Precision Cell Technology, Subang Jaya, Malaysia) and was routinely cultured with growth medium for non-tumorigenic human cells [low-glucose Dulbecco's Modified Eagle's Medium (DMEM; Gibco; Thermo Fisher Scientific, Inc., Waltham, MA, USA) supplemented with $10 \%$ fetal bovine serum (FBS), 100 units/ml penicillin and $100 \mathrm{mg} / \mathrm{ml}$ streptomycin with stable glutamine and sodium pyruvate], whereas the MCF-7 cell line was cultured using the growth medium for tumorigenic human cells [high-glucose DMEM supplemented with $10 \%$ FBS, 100 units $/ \mathrm{ml}$ penicillin and $100 \mathrm{mg} / \mathrm{ml}$ streptomycin]. Occasionally, an optional supplement of 1X MycoKill (PAA Laboratories; GE Healthcare Life Sciences, Chalfont, UK) and an antibiotic cocktail were added to the two growth media to prevent mycoplasma and fungal contaminations, respectively. The cell lines were maintained at $37^{\circ} \mathrm{C}$ in a humidified atmosphere of $5 \%(\mathrm{v} / \mathrm{v}) \mathrm{CO}_{2}$. The growth media for the BMSCs and MCF-7 cells were changed every three to four days. Cell lines were subsequently subcultured and maintained for adhesive and non-adhesive stem-and-cancer cell interaction, as described below (Fig. 2).

Analysis of the adhesive interaction of MCF-7 cells with pioglitazone- and/or rosiglitazone-pretreated BMSC feeder layers. The adhesive interaction or direct co-culture of MCF-7 cells with BMSCs pretreated with growth media supplemented with pioglitazone and/or rosiglitazone (both Sigma-Aldrich, St. Louis, MO, USA) was performed by seeding $1.0 \times 10^{3} \mathrm{BMSCs} / \mathrm{ml}$ per well in a four-well chamber slide. The cells were allowed to adhere overnight. The low-glucose DMEM growth medium for the BMSCs was changed every three to four days, when the cells reached $90 \%$ confluence. The BMSCs were subsequently incubated in growth medium supplemented with pioglitazone and/or rosiglitazone for one week. Pioglitazone $(40 \mu \mathrm{M})$ or rosiglitazone $(40 \mu \mathrm{M})$, or pioglitazone $(20 \mu \mathrm{M})+$ rosiglitazone $(20 \mu \mathrm{M})$, which modified the BMSCs but did not significantly decrease their viability, was added to the growth medium to treat the BMSCs. Dimethylsulfoxide (DMSO; Bio Basic Canada, Inc., Markham, ON, Canada) was used as a control in the present study, since it has been demonstrated to exert no growth-reducing effect on MCF-7 cells. The incubated cells were observed on a daily basis, and the growth medium supplemented with pioglitazone and/or rosiglitazone was changed every three to four days. After one week, the growth medium supplemented with pioglitazone and/or rosiglitazone was removed. The BMSC feeder layer that had been incubated with the growth media supplemented with pioglitazone and/or rosiglitazone was carefully washed several times with pre-warmed phosphate-buffered saline (PBS). Once the growth medium supplemented with pioglitazone and/or rosiglitazone had been removed, the BMSC feeder layer was referred to as the pioglitazone- and/or rosiglitazone-pretreated BMSC feeder layer. A suspension of MCF-7 cells (10 cells/well) was added to the pretreated BMSC feeder layer. Cultured MCF-7 cells alone were used as a control in the present experiment. Following one week of co-culture, the morphology, number and size of the MCF-7 colonies that were formed on the pretreated BMSC feeder layer were monitored and quantified using Oil Red O staining under a Motic AE31 inverted microscope (Motic Intruments, Inc., Richmond, BC, Canada). The size (diameter) of each MCF-7 colony on the pretreated BMSC feeder layer was measured at a magnification of $\mathrm{x} 40$ using the inverted microscope. The co-culture was repeated in at least two independent experiments to ensure the reproducibility of the results.

Analysis of the non-adhesive interaction of $\mathrm{MCF}-7$ cells with pioglitazone- and/or rosiglitazone-pretreated BMSC conditioned medium. The non-adhesive interaction or indirect co-culture of MCF-7 cells with the conditioned medium of BMSCs pretreated with pioglitazone and/or rosiglitazone was performed by seeding $1.0 \times 10^{4} \mathrm{BMSCs} / \mathrm{ml}$ per well in a six-well plate. The cells were allowed to adhere overnight. The low-glucose DMEM growth medium for the BMSCs was changed every three to four days. Once the cells had reached $90 \%$ confluence, the growth medium used to maintain the BMSCs was removed, and the BMSC feeder was subsequently added with the growth media supplemented with pioglitazone and/or rosiglitazone. The pioglitazone- and/or rosiglitazone-containing growth medium used to pretreat the BMSCs was changed every three to four days. After one week, the growth medium supplemented with pioglitazone and/or rosiglitazone was removed. Subsequently, the pioglitazoneand/or rosiglitazone-pretreated BMSC feeder layer was carefully washed several times with pre-warmed PBS. Fresh growth medium was subsequently added to the pretreated BMSCs, and the pretreated BMSC feeder layer was incubated in fresh growth medium for one week. Following one week of incubation, the growth medium, which is now referred to as conditioned medium for all culture conditions, was collected in $15 \mathrm{ml}$ falcon tubes (BD Biosciences, San Jose, CA, USA). The conditioned medium was subsequently centrifuged using an Eppendorf 5804R (Eppendorf, Germany) at maximum speed $(15,000 \mathrm{x} \mathrm{g})$ for $20 \mathrm{~min}$ at $4^{\circ} \mathrm{C}$ to precipitate any particles or cell debris in the medium, and the supernatants were collected and used for the MCF-7 cell incubation in the analysis of non-adhesive interaction, as described below. The conditioned medium was also used for immunoassays, as described below. For the non-adhesive interaction, the MCF-7 cells were seeded $\left(1.0 \times 10^{3}\right.$ cells $/ \mathrm{ml}$ per well) and maintained in high-glucose DMEM growth medium in a 12-well plate. Following $24 \mathrm{~h}$ of incubation, the old growth medium was removed, and the collected conditioned medium was added to the MCF-7 cells. 
The MCF-7 cells were incubated in the conditioned medium for one week, and the incubation of the MCF-7 cells with each conditioned medium was performed in triplicate. Following one week of incubation, the MCF-7 cells were trypsinized using $0.25 \%$ trypsin-EDTA containing phenol red, and the proliferation rate of the cells was quantified using a Trypan Blue Exclusion assay using $0.4 \%$ (w/v) Trypan Blue solution (Gibco). The co-culture was repeated in at least two independent experiments to ensure the reproducibility of the results.

Immunoassay of soluble growth factors in conditioned medium of BMSCs pretreated with pioglitazone and/or rosiglitazone. The levels of FGF4, CCL2, CCL5, IL-6, vascular endothelial growth factor (VEGF) and transforming growth factor $\beta$ (TGF $\beta$ ) in the conditioned medium of BMSCs pretreated with pioglitazone and/or rosiglitazone were determined using RayBio $^{\circledR}$ enzyme-linked immunosorbent assay (ELISA) kits (RayBiotech, Inc., Norcross, GA, USA). In these kits, antibodies specific for the proteins were pre-coated onto microtiter plates. The samples (conditioned medium) were subsequently added to the wells and allowed to react with the bound antibody for $2.5 \mathrm{~h}$ at room temperature. The unbound substances were washed away with wash solution, according to the manufacturer's protocol. Subsequently, an enzyme-linked antibody specific to the protein in the conditioned medium was added to the wells. The antibody was incubated with the target protein for $1 \mathrm{~h}$. Following a further washing step, substrate solution was added to the wells for color development. Development of the color was proportional to the quantity of protein present in the samples. The color intensity was subsequently measured using an ELISA reader at a wavelength of $450 \mathrm{~nm}$, and the level of each specific protein in the conditioned medium was calculated. The ELISA was performed in triplicate and repeated in at least two independent experiments.

Analysis of the levels of FGF4 transcript in BMSCs pretreated with pioglitazone and/or rosiglitazone. The total RNA of the pretreated BMSCs was extracted using the RNeasy $^{\text {TM }}$ Total RNA kit (Qiagen, Inc., Valencia, CA, USA). The integrity of the extracted total RNA was confirmed by $1 \%$ agarose gel electrophoresis at $90 \mathrm{~V}$ for $35 \mathrm{~min}$, and the purity, as well as the concentration of extracted total RNA, was measured using a Nanodrop 2000c spectrophotometer (Nanodrop; Thermo Fisher Scientific, Inc.) at 260/280 nm. Subsequently, $1.0 \mu \mathrm{g}$ RNA was reverse-transcribed into cDNA using a commercially available Revert-Aid First-Strand cDNA Synthesis kit (Applied Biosystems; Thermo Fisher Scientific, Inc.), and this cDNA was used to analyze the level of FGF4 transcript in the pretreated BMSCs using real-time (RT)-quantitative (q)PCR. During this process, the cDNA that was reverse-transcribed from the extracted total RNA of pioglitazone- and/or rosiglitazone-pretreated adipose tissue-derived mesenchymal stem cells (ATSCs) was also used to analyze the level of the FGF4 transcript by RT-qPCR. Primers specific to the genes were designed using Primer Express software version 2.0 (Applied Biosystems) (Table I), and RT-qPCR was performed using a Rotor-Gene 600 PCR system (Qiagen, Inc.), according to the manufacturer's protocol. The reactions were performed in a total volume of $25 \mu 1$ in optical reaction tubes that included Power SYBR ${ }^{\circledR}$ Green

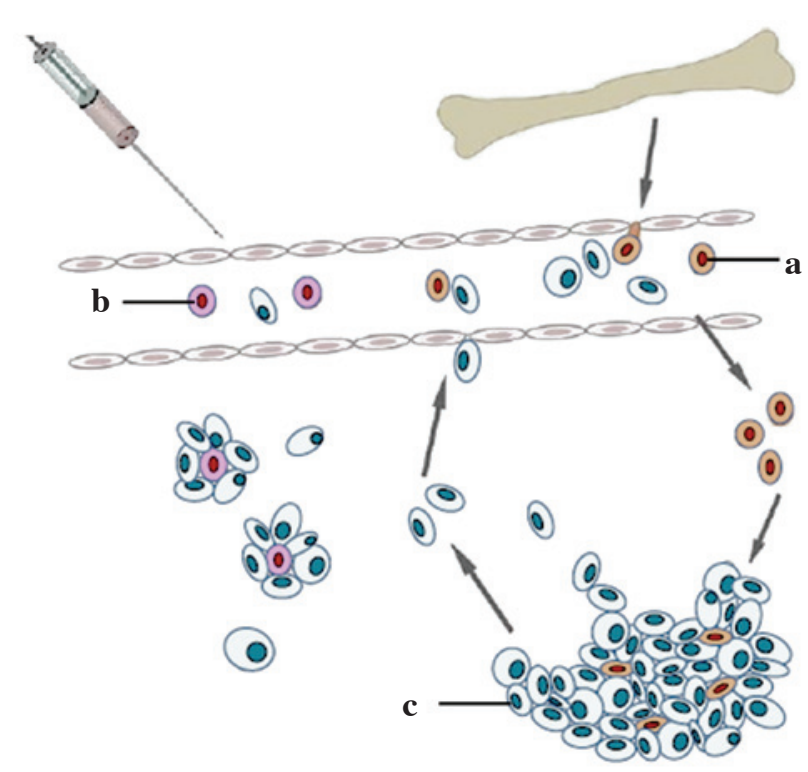

Figure 1. Schematic overview of the role of BMSCs (labelled 'a') and pioglitazone- and/or rosiglitazone-pretreated BMSCs (labelled ' $b$ ') in the interaction of stem and cancer cells. The cancer cells are labelled 'c'. BMSCs increase the growth (colony size) and proliferation rate (colony number) of cancer cells. The hypothesis of the present study was to inject pretreated BMSCs into the cancerous site or bloodstream of a cancer patient, in an effort to reduce the growth and proliferation rate of the cancer cells as they interact adhesively and non-adhesively with the pretreated BMSCs. BMSCs, bone marrow-derived mesenchymal stem cells.

Master mix (Applied Biosystems), $900 \mathrm{nM}$ each primer and cDNA, as prepared above. The reaction program was subsequently initiated at $95^{\circ} \mathrm{C}$ for 10 min to activate the enzyme, and this step was followed by 40 cycles of denaturation at $95^{\circ} \mathrm{C}$ for $15 \mathrm{sec}$ and primer annealing combined with extension at $60^{\circ} \mathrm{C}$ for $1 \mathrm{~min}$. The $\mathrm{Ct}$ value of the gene in each unknown sample was normalized to that of $\beta$-actin, and the associated expression level of the gene and the fold change in gene expression were calculated, according to the manufacturer's protocol. RT-qPCR was performed in triplicate, and repeated in at least two independent experiments.

Analysis of the effect of an FGF4-neutralizing antibody on the non-adhesive interaction of MCF-7 cells with BMSCs. The effect of a FGF4-neutralizing antibody on the non-adhesive interaction of MCF-7 cells with BMSCs was analyzed by seeding $5.0 \times 10^{3} \mathrm{BMSCs} / \mathrm{ml}$ per well in a 24 -well plate. The cells were allowed to adhere until $70 \%$ confluence had been reached. At this point, the old growth medium used to maintain the BMSCs was subsequently removed, and the BMSC feeder layer was added with fresh growth medium. The fresh growth medium contained 6,10 or $14 \mu \mathrm{g} / \mathrm{ml}$ anti-human FGF4-neutralizing antibody (R\&D Systems, Inc., Minneapolis, MN, USA). The incubation of the stem cells with fresh growth medium without FGF4-neutralizing antibody was used as a control in the present study. A polycarbonate Transwell cell insert with a membrane pore size of $8.0 \mu \mathrm{m}$ was subsequently attached to each well of the plate as a transwell configuration. A $100 \mu \mathrm{l}$ suspension of MCF-7 cells ( $\sim 100$ cells/well) was subsequently added onto the cell insert. The cancer cells were allowed to interact with the stem cells in the respective growth medium with or without the neutralizing antibody for 
Table I. Primers used for real time-quantitative PCR.

\begin{tabular}{|c|c|c|}
\hline Gene(s) & Sequence(s) & Amplicon (bp) \\
\hline FGF4 & $\begin{array}{l}\text { Forward: 5'-CAACTACAACGCCTACGAGTCCTA-3' } \\
\text { Reverse: 5'-CCTTCTTGGTCTTCCCATTCTTG-3' }\end{array}$ & 77 \\
\hline $\mathrm{Ki}-67^{\mathrm{a}}$ & $\begin{array}{l}\text { Forward: 5'-AACTATCCTCGTCTGTCCCAACAC-3' } \\
\text { Reverse: 5'-CGGCCATTGGAAAGACAGAT-3' }\end{array}$ & 106 \\
\hline $\mathrm{PCNA}^{\mathrm{b}}$ & $\begin{array}{l}\text { Forward: 5'-AGAAGGTGTTGGAGGCACTCA-3' } \\
\text { Reverse: 5'-GGTTTACACCGCTGGAGCTAA-3' }\end{array}$ & 72 \\
\hline$\beta$-actin & $\begin{array}{l}\text { Forward: 5'-CATTGCCGACAGGATGCA-3' } \\
\text { Reverse: 5'-CCGATCCACACGGAGTACTTG-3' }\end{array}$ & 102 \\
\hline
\end{tabular}

\section{$\underline{\text { Adhesive interaction } \quad \text { Non-adhesive interaction }}$}

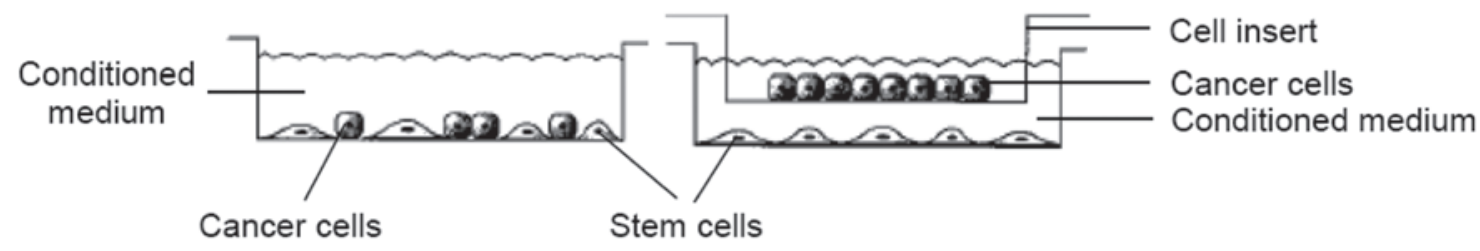

Figure 2. Schematic overview of the adhesive and non-adhesive interactions. Adhesive interactions were defined as the growth of cancer cells on the BMSC feeder layer, where direct physical cell-cell interactions occur. Non-adhesive interactions were defined as the incubation of cancer cells with the BMSC conditioned medium, whereby both cell populations interacted in different compartments (insert and well) and communicated via growth factors in the conditioned medium through the pores in the cell membrane of the insert. BMSCs, bone marrow-derived mesenchymal stem cells.

one week. The growth media was changed once during the incubation period for the stem-and-cancer cell interaction. After one week of incubation, to quantify the exact proliferation rate of incubated MCF-7 cells, the MCF-7 cells were trypsinized, as previously described, and subjected to RNA extraction and cDNA synthesis, and the resulting cDNA was subsequently used to analyze the levels of Ki-67 and proliferating cell nuclear antigen (PCNA) transcripts by RT-qPCR, as described above. The co-culture was repeated in at least two independent experiments to ensure the reproducibility of the results.

Statistical analysis. All the graphs and statistical calculations were generated and performed using GraphPad 6.01 software (GraphPad Software, Inc., La Jolla, CA, USA). Despite the adhesive nature of the co-culture, the majority of the experiments were performed in triplicate, and repeated several times independently to confirm the reproducibility of the results. Data are expressed as the mean \pm standard deviation. $\mathrm{P}<0.05$ was taken to indicate a statistically significant difference.

\section{Results}

Adhesive interaction of MCF-7 cells grown on pioglitazoneand/or rosiglitazone-pretreated BMSC feeder layers. For the adhesive interaction, the growth and proliferation rate of
MCF-7 cells on the pioglitazone- and/or rosiglitazone-pretreated BMSC feeder layers were measured by the colony size (growth) and the colony number (proliferation rate) of the cancer cells, respectively. In general, compared with the MCF-7 cells grown on plastic only for one week (Fig. 3A), the growth of the MCF-7 cells (colony size) was observed to be greater and more diffuse when the cancer cells were co-cultured on the feeder layer of BMSCs pretreated with DMSO alone (control; Fig. 3B). A similar phenomenon was observed when the MCF-7 cells were grown on the feeder layers of BMSCs pretreated with $40 \mu \mathrm{M}$ pioglitazone (Fig. 3C) and $40 \mu \mathrm{M}$ rosiglitazone (Fig. 3D). The overall size of the MCF-7 colonies formed on the feeder layer of BMSCs pretreated with DMSO alone was assigned a value of $100 \%$ in the present study (control feeder layer). The MCF-7 colonies formed on the feeder layer of BMSCs pretreated with $40 \mu \mathrm{M}$ pioglitazone and $40 \mu \mathrm{M}$ rosiglitazone were $\sim 113.0$ to $115.3 \%$, and $\sim 96.2$ to $104.1 \%$, respectively, compared with the MCF-7 colonies formed on the control feeder layer. The appearance of these MCF-7 colonies was also revealed to be single cells, without evidence of direct cell-to-cell contact. However, when MCF-7 cells were co-cultured on feeder layers of BMSCs that were pretreated with $20 \mu \mathrm{M}$ pioglitazone $+20 \mu \mathrm{M}$ rosiglitazone, the overall size of the MCF-7 colonies that were formed on the feeder layer was markedly decreased to $\sim 62.5$ to $77.6 \%$ (Fig. 3E) compared with the control feeder layer. The two small colonies in the field of view are not comparable to 
A

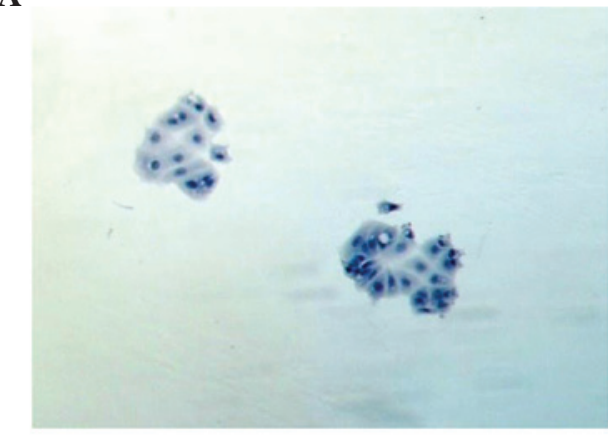

C

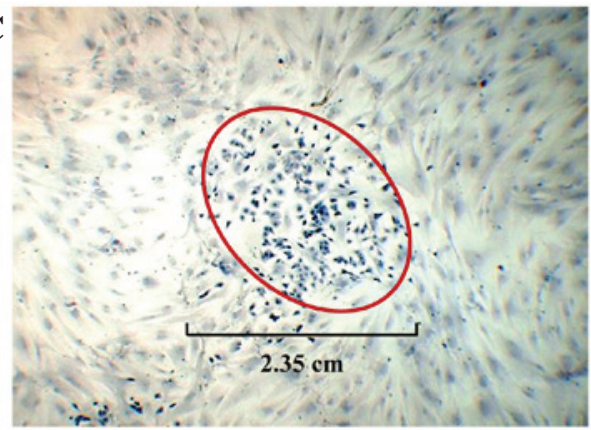

B

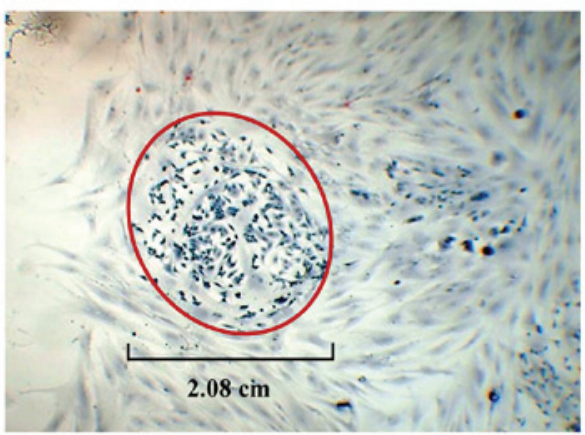

D

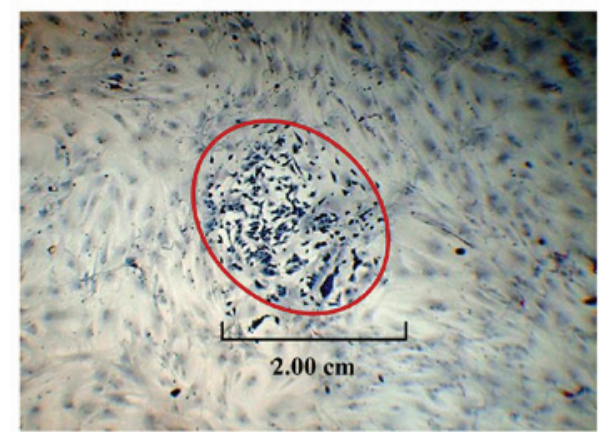

$\mathbf{E}$

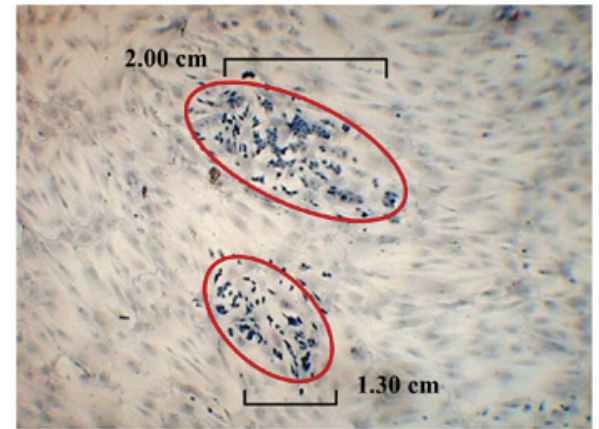

Figure 3. Colony formation potential of MCF-7-cells cultured with or without BMSCs. (A) The formation of MCF-7 colonies on a culture flask (without BMSCs) is shown. (B-E) The formation of MCF-7 colonies on feeder layers of BMSCs pretreated with (B) dimethylsulfoxide (control), (C) $40 \mu \mathrm{M} \mathrm{pioglitazone,}$ (D) $40 \mu \mathrm{M}$ rosiglitazone and (E) $20 \mu \mathrm{M}$ pioglitazone $+20 \mu \mathrm{M}$ rosiglitazone are shown. A magnification of x100 was used for (A), whereas a magnification of $\mathrm{x} 40$ was used for (B-E). BMSCs, bone marrow-derived mesenchymal stem cells.

a large colony, since there is an appreciable distance between the areas of the two colonies. Thus, BMSCs pretreated with $20 \mu \mathrm{M}$ pioglitazone $+20 \mu \mathrm{M}$ rosiglitazone may have the potential to reduce the growth of MCF-7 cells.

In addition to the growth of MCF-7 cells, a similar phenomenon was observed on the proliferation rate of MCF-7 cells (colony number). When MCF-7 cells were co-cultured on the feeder layer of BMSCs that were pretreated with a single type of TZD drug, or with the two of them, the number of MCF-7 colonies that were formed on the BMSC feeder layer was markedly decreased compared with the number of colonies that were formed on the control feeder layer (Fig. 4). The number of MCF-7 colonies formed was decreased to $69.1 \%$ by co-culturing with the feeder layer of BMSCs pretreated with $40 \mu \mathrm{M}$ pioglitazone $(\mathrm{P}<0.05)$, whereas the number decreased to $55.8 \%$ by co-culturing with the feeder layer of BMSCs pretreated with $40 \mu \mathrm{M}$ rosiglitazone $(\mathrm{P}<0.01)$, and to $59.2 \%$ by co-culturing with the feeder layer of BMSCs pretreated with $20 \mu \mathrm{M}$ pioglitazone $+20 \mu \mathrm{M}$ rosi- glitazone $(\mathrm{P}<0.01)$. The calculation was based on the number of MCF-7 colonies that were formed on the feeder layer of BMSCs pretreated with DMSO, which was set to $100 \%$ in the present study (control feeder layer). Thus, BMSCs pretreated with one, or a combination, of the TZD drugs may have the potential to reduce the proliferation rate of cancer cells.

Non-adhesive interaction of MCF-7 cells grown in pioglitazone- and/or rosiglitazone-pretreated BMSC conditioned medium. Unlike the experiment investigating adhesive interaction, only the proliferation rate was determined in the case of non-adhesive interaction. The non-adhesive interaction of MCF-7 cells with the conditioned medium of BMSCs increased the proliferation rate of the cancer cells by $\sim 16.6 \%$ compared with the proliferation rate of the cancer cells that were incubated in the growth medium only (Fig. 5). This phenomenon indicated that the increase in the proliferation rate of cancer cells cannot be correlated with a direct physical stem-and-cancer cell interaction, since similar findings were observed for the adhesive 


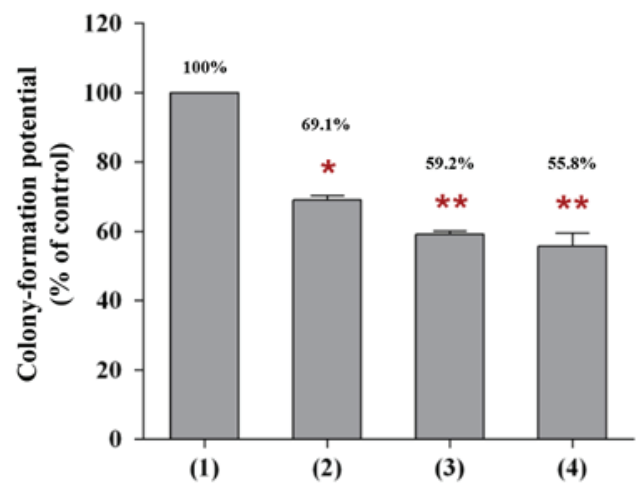

Figure 4. Proliferation rate of MCF-7 cells was assessed using a traditional clonogenic assay. The number of MCF-7 colonies formed was determined on the feeder layer of (1) BMSCs pretreated with DMSO only, (2) BMSCs pretreated with $40 \mu \mathrm{M}$ pioglitazone, (3) BMSCs pretreated with $20 \mu \mathrm{M}$ pioglitazone $+20 \mu \mathrm{M}$ rosiglitazone, and (4) BMSCs pretreated with $40 \mu \mathrm{M}$ rosiglitazone. All the co-cultures were incubated for one week Data are expressed as the means \pm standard deviation from three separate experiments. One-way analysis of variance was used to compare the proliferation rate of BMSCs pretreated with DMSO only (control) to the BMSCs pretreated with thiazolidinedione(s). ${ }^{*} \mathrm{P}<0.05,{ }^{* * *} \mathrm{P}<0.01$, compared with the control. BMSCs, bone marrow-derived mesenchymal stem cells; DMSO, dimethylsulfoxide.

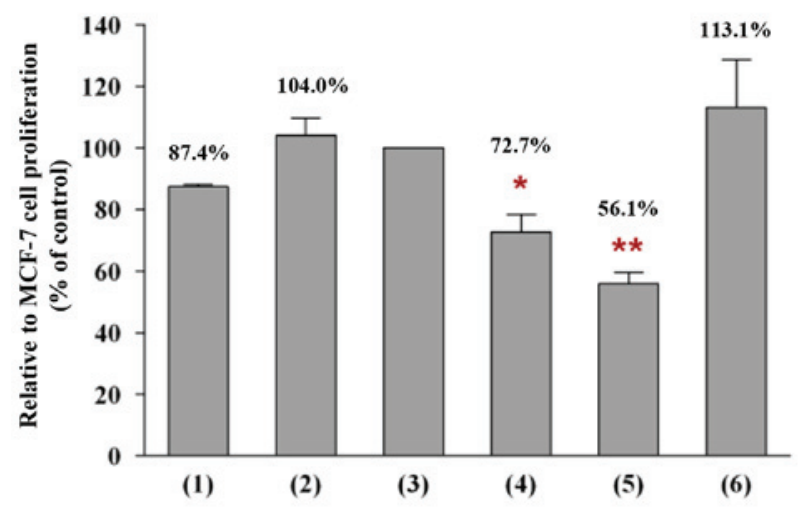

Figure 5. Proliferation rate of MCF-7-cells incubated in the conditioned medium of non-pretreated and pretreated BMSCs for one week. Results from the incubation of MCF-7-cells with (1) growth medium only, (2) conditioned medium of BMSCs only, (3) conditioned medium of BMSCs pretreated with DMSO, (4) conditioned medium of BMSCs pretreated with $40 \mu \mathrm{M}$ pioglitazone, (5) conditioned medium of BMSCs pretreated with $20 \mu \mathrm{M}$ pioglitazone $+20 \mu \mathrm{M}$ rosiglitazone, and (6) conditioned medium of BMSCs pretreated with $40 \mu \mathrm{M}$ rosiglitazone are shown. A total of 100 cells was used as the input cells for the incubation. The proliferation rate of MCF-7-cells incubated in the conditioned medium of BMSCs pretreated with DMSO (control) was considered as $100 \%$ in the present study. Data are expressed as the means \pm standard deviation from three separate experiments. One-way analysis of variance was used to compare the proliferation rate of BMSCs pretreated with DMSO only (control) to the BMSCs pretreated with thiazolidinedione(s). ${ }^{*} \mathrm{P}<0.05,{ }^{* *} \mathrm{P}<0.01$ compared with the control. BMSCs, bone marrow-derived mesenchymal stem cells; DMSO, dimethylsulfoxide.

and the non-adhesive interaction conditions. Furthermore, the incubation of MCF-7 cells with BMSC conditioned medium caused the cells to convert from growing in clusters into appearing as single cells, without evidence of direct cell-to-cell contact. However, the incubation of MCF-7 cells with the conditioned medium of BMSCs pretreated with pioglitazone and/or rosiglitazone reduced the proliferation rate of the cancer cells.
Incubation of MCF-7 with the conditioned medium of BMSCs pretreated with pioglitazone and/or rosiglitazone decreased the proliferation rate of the cancer cells compared with the incubation of MCF-7 cells in the conditioned medium of BMSCs pretreated with DMSO only (100\%; control). The present study determined that the proliferation rate of $\mathrm{MCF}-7$ cells was decreased to $72.7 \%$ $(\mathrm{P}<0.05)$ when the cancer cells were incubated in the conditioned medium of BMSCs pretreated with $40 \mu \mathrm{M}$ pioglitazone (Fig. 5). The growth reduction effect on the proliferation rate was observed to be more potent when the MCF-7 cells were incubated in the conditioned medium of BMSCs pretreated with $20 \mu \mathrm{M}$ pioglitazone $+20 \mu \mathrm{M}$ rosiglitazone, which decreased the proliferation rate of MCF-7 cells to $56.1 \%$ ( $\mathrm{P}<0.01)$. However, the incubation of MCF-7 cells with the conditioned medium of BMSCs pretreated with $40 \mu \mathrm{M}$ rosiglitazone did not reveal any growth reduction effect on the proliferation rate of cancer cells. This finding indicates that the growth reduction effect on cancer cells may be caused by a reduced secretion of specific soluble growth factors by pretreated BMSCs into the conditioned medium.

Levels of soluble growth factors in the conditioned medium of BMSCs pretreated with pioglitazone and/or rosiglitazone. The present study identified that the soluble growth factors secreted by pretreated BMSCs in the conditioned medium differed, depending on the TZD treatment (Fig. 6). Decreased levels of FGF4 were first detected in the conditioned medium of BMSCs that were pretreated with pioglitazone and/or rosiglitazone, as determined by ELISA. The present study revealed that the levels of FGF4 in the conditioned medium of BMSCs pretreated with $40 \mu \mathrm{M}$ pioglitazone and $40 \mu \mathrm{M}$ rosiglitazone were $34.0 \%(\mathrm{P}<0.001)$ and $18.8 \%(\mathrm{P}<0.001)$, respectively, compared with the level of FGF4 in the conditioned medium of BMSCs pretreated with DMSO (100\%; control). When the BMSCs were pretreated with a combination of pioglitazone and rosiglitazone, a more marked reduction in the level of FGF4 in the conditioned medium of pretreated BMSCs was observed. The level of FGF4 in the conditioned medium of BMSCs pretreated with $20 \mu \mathrm{M}$ pioglitazone $+20 \mu \mathrm{M}$ rosiglitazone was only $14.8 \%(\mathrm{P}<0.001)$ compared with the control. The marked reduction in the level of FGF4 may contribute to the potent decrease in the proliferation rate of MCF-7 cells. Indeed, the levels of CCL5 and IL-6 were also identified to be significantly higher in the conditioned medium of BMSCs pretreated with $40 \mu \mathrm{M}$ rosiglitazone $(\mathrm{P}<0.05)$, and the level of VEGF was significantly higher in the conditioned medium of BMSCs pretreated with $40 \mu \mathrm{M}$ pioglitazone $(\mathrm{P}<0.05)$, indicating the negative implications of pioglitazone use. The high levels of CCL5 and IL-6 in the conditioned medium of BMSCs pretreated with $40 \mu \mathrm{M}$ rosiglitazone may explain why the proliferation rate of MCF-7 cells did not decrease when the cancer cells were incubated in the conditioned medium.

Levels of FGF4 transcript in BMSCs pretreated with pioglitazone and/or rosiglitazone. A reduction in the levels of FGF4 transcript in BMSCs pretreated with pioglitazone and/or rosiglitazone by RT-qPCR was observed (Fig. 7). However, the reduction in the levels of FGF4 transcript in BMSCs pretreated with pioglitazone and/or rosiglitazone was not statistically significant compared with the levels observed in the BMSCs pretreated with DMSO only. The level of FGF4 transcript in 
A

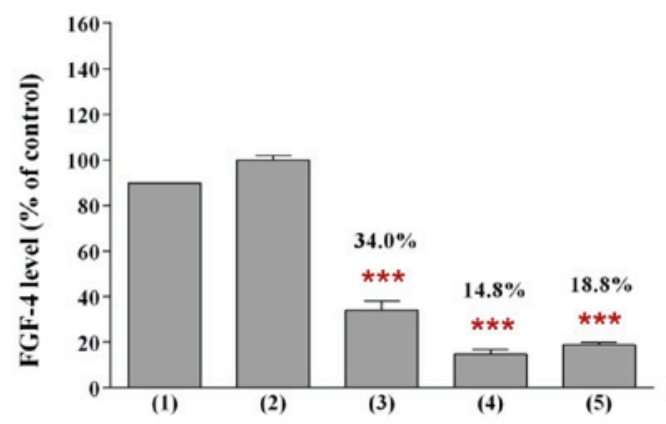

C

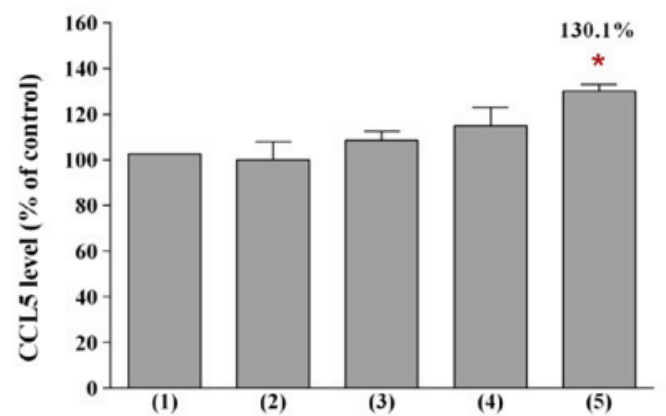

$\mathbf{E}$

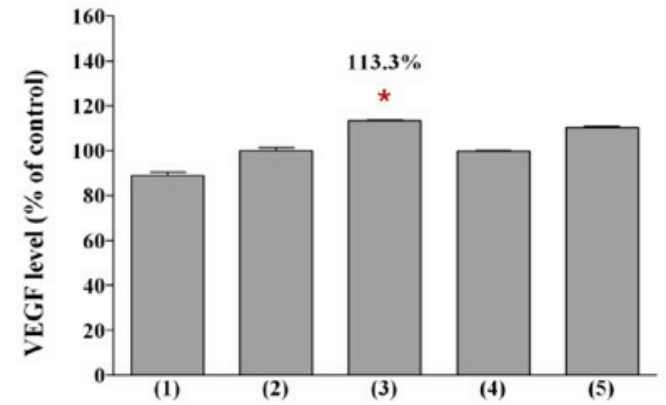

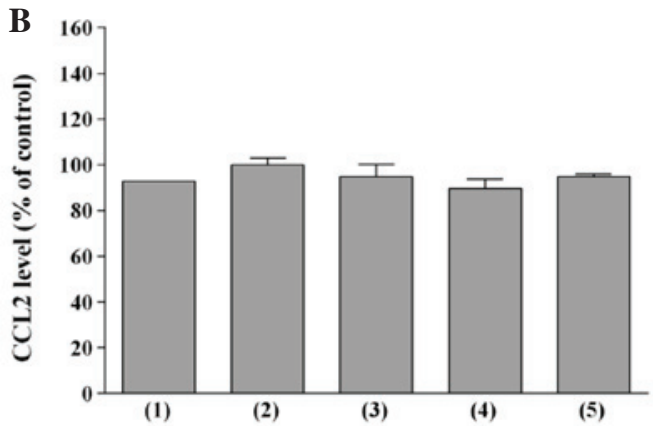

D

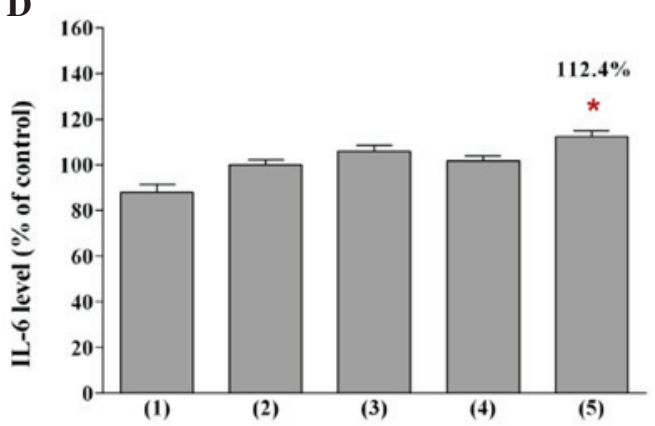

F

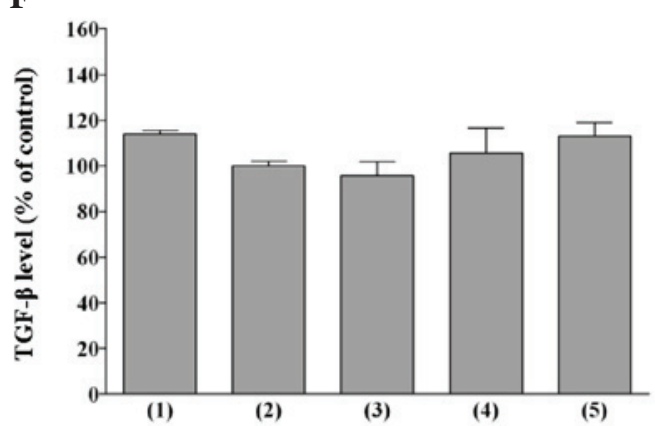

Figure 6. Levels of (A) FGF4, (B) CCL2, (C) CCL5, (D) IL-6, (E) VEGF and (F) TGF- $\beta$ in the conditioned medium of BMSCs pretreated with pioglitazone and/or rosiglitazone. The experimental conditions were: (1) conditioned medium of BMSCs, (2) conditioned medium of BMSCs pretreated with DMSO, (3) conditioned medium of BMSCs pretreated with $40 \mu \mathrm{M}$ pioglitazone, (4) conditioned medium of BMSCs pretreated with $20 \mu \mathrm{M}$ pioglitazone $+20 \mu \mathrm{M}$ rosiglitazone, and (5) conditioned medium of BMSCs pretreated with $40 \mu \mathrm{M}$ rosiglitazone. The levels of these soluble growth factors in the conditioned medium were assayed by enzyme-linked immunosorbent assay. The levels of soluble growth factors in the conditioned medium of BMSCs pretreated with DMSO were defined as $100 \%$. Data are expressed as the means \pm standard deviation from three separate experiments. One-way analysis of variance was used to compare the levels of soluble growth factor in BMSCs pretreated with DMSO only (control) with the BMSCs pretreated with the thiazolidinedione(s). ${ }^{*} \mathrm{P}<0.05,{ }^{* * *} \mathrm{P}<0.001$ compared with the control. FGF4, fibroblast growth factor 4; CCL2/5, chemokine (C-C motif) ligand-2/5; IL-6, interleukin-6; VEGF, vascular endothelial growth factor; TGF- $\beta$, transforming growth factor- $\beta$; BMSCs, bone marrow-derived mesenchymal stem cells; DMSO, dimethylsulfoxide.

the BMSCs pretreated with $20 \mu \mathrm{M}$ pioglitazone $+20 \mu \mathrm{M}$ rosiglitazone was only $88.78 \%$ compared with the control (100\%). This phenomenon indicated that a reduction in the expression level of the FGF4 protein only contributed to the growth reduction effect on MCF-7 cells when the cancer cells interacted with the pretreated stem cells.

Effect of an FGF4-neutralizing antibody on the non-adhesive interaction of MCF-7 cells with BMSCs. The present study revealed reduced levels of $\mathrm{Ki}-67$ and PCNA transcripts in MCF-7 cells that interacted non-adhesively with BMSCs in growth medium containing the FGF4-neutralising antibody (Fig. 8). The levels of the transcripts in MCF-7 cells that underwent the identical interaction with BMSCs in growth medium without the antibody was set to $100 \%$ (control). The reduction in the levels of $\mathrm{Ki}-67$ and PCNA transcripts in the MCF-7 cells that had interacted with the BSMCs was statisti- cally significant. In fact, the levels of the two transcripts in MCF-7 cells that interacted with BMSCs in growth medium containing $6 \mu \mathrm{g} / \mathrm{ml} \mathrm{FGF4-neutralizing} \mathrm{antibody} \mathrm{were} 80 \%$ and $42 \%(\mathrm{P}<0.01)$, respectively, compared with the control $(100 \%)$. When the cancer cells that had interacted with BMSCs were incubated with medium containing 10 and $14 \mu \mathrm{g} / \mathrm{ml}$ FGF4-neutralizing antibody, the levels of the Ki-67 and PCNA transcripts were reduced to $31 \%(\mathrm{P}<0.001)$ and $28 \%$ $(\mathrm{P}<0.001)$, and to $22 \%(\mathrm{P}<0.001)$ and $27 \%(\mathrm{P}<0.001)$, respectively, compared with the identical control. Since Ki-67 and PCNA are widely used as proliferation-associated markers, this phenomenon indicated that the neutralization of FGF4 secreted from the BMSCs in growth medium effected by the FGF4-neutralizing antibody reduced the proliferation rate of the MCF-7 cells. Thus, the decrease in the proliferation rate of MCF-7 cells was likely to have been caused by the reduction in the level of FGF4. 


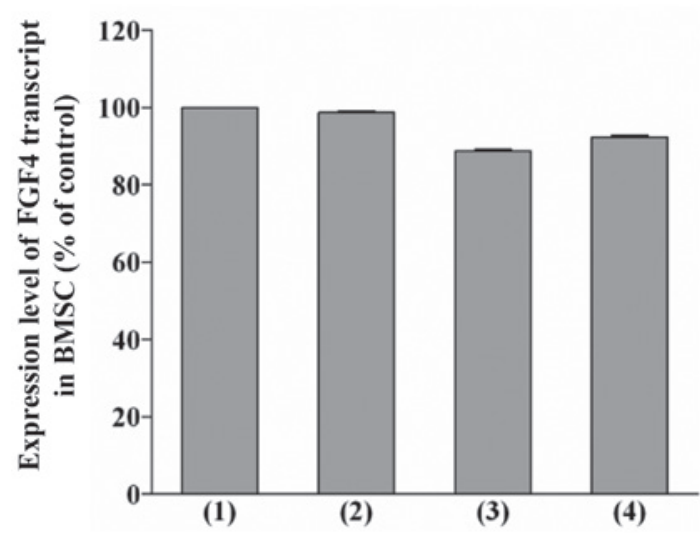

Figure 7.Level of FGF4 transcript in BMSCs pretreated with pioglitazone and/or rosiglitazone for one week. The experimental conditions were: (1) BMSCs pretreated with DMSO only, (2) BMSCs pretreated with $40 \mu \mathrm{M}$ pioglitazone, (3) BMSCs pretreated with $20 \mu \mathrm{M}$ pioglitazone $+20 \mu \mathrm{M}$ rosiglitazone, and (4) BMSCs pretreated with $40 \mu \mathrm{M}$ rosiglitazone for one week. The gene transcript levels were analyzed using real-time quantitative polymerase chain reaction. Each datum was calculated as the percentage of the level of the FGF4 transcript compared with the level of $\beta$-actin in the pretreated BMSCs, relative to the $\%$ of control. Data are shown as the means \pm standard deviation from three independent experiments. One-way analysis of variance was used to compare the level of FGF4 transcript in BMSCs pretreated with DMSO only (control) to the BMSCs pretreated with thiazolidinedione(s). BMSCs, bone marrow-derived mesenchymal stem cells; FGF4, fibroblast growth factor 4; DMSO, dimethylsulfoxide.

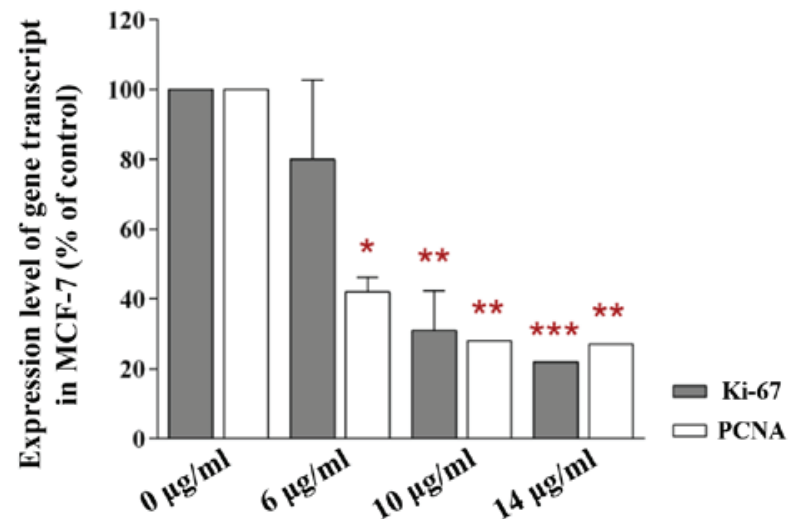

Figure 8. Levels of Ki-67 and PCNA transcripts in MCF-7 cells that interacted non-adhesively with BMSCs in growth medium containing different concentrations $(\mu \mathrm{g} / \mathrm{ml})$ of the FGF4-neutralizing antibody. The gene transcripts were analyzed using real-time quantitative polymerase chain reaction Each datum was calculated as the percentage of the level of the gene transcript compared with the level of $\beta$-actin in the pretreated BMSCs, relative to the $\%$ of control. The levels are shown as the means \pm standard deviation from three independent experiments. One-way analysis of variance was used to compare the level of each gene transcript in MCF-7 cells that interacted with BMSCs in medium with or without the FGF4-neutralizing antibody for one week. ${ }^{*} \mathrm{P}<0.05,{ }^{* *} \mathrm{P}<0.01,{ }^{* * *} \mathrm{P}<0.001$ compared with the control. BMSCs, bone marrow-derived mesenchymal stem cells; FGF4, fibroblast growth factor 4; PCNA, proliferating cell nuclear antigen.

\section{Discussion}

BMSCs have been previously shown to secrete soluble growth factors into conditioned medium (6-14). Thus, pretreated BMSCs may be used to alter the growth and proliferation rate of MCF-7 cells via changes in the levels of soluble growth factors secreted by the pretreated BMSCs into the conditioned medium. In the present study, it is proposed that the inhibition of the MCF-7 growth and proliferation rate was likely to have been due to a reduction in the secretion of specific soluble growth factors by the pretreated BMSCs, regardless of the presence or absence of a direct physical stem-and-cancer cell interaction between the two cell lines. The level of soluble growth factors in the conditioned medium secreted by pretreated BMSCs were likely to exert a growth reduction effect, which prohibited the growth and proliferation rate of the MCF-7 cells. The soluble growth factors assessed in the present study, including FGF4, CCL2, CCL5 (also termed RANTES), IL-6, VEGF and TGF- $\beta$, have been previously reported to exhibit proliferation-associated effects on cancer cell growth (6-14). However, a reduction in FGF4 was only identified in the conditioned medium of BMSCs pretreated with pioglitazone and/or rosiglitazone.

Pioglitazone and rosiglitazone exhibit a similar safety profile and effect on cancer cells. Consistently with these findings, the present study identified high levels of soluble growth factors, which are usually increased in the inflammatory response and during cancer cell invasion, in the conditioned medium of BMSCs pretreated with pioglitazone and rosiglitazone. VEGF, which is known to exert an important role in angiogenesis, was also present at markedly higher levels in the conditioned medium of BMSCs pretreated with $40 \mu \mathrm{M}$ pioglitazone, and therefore this could explain the unsuitability of pioglitazone for pretreatment of the BMSCs, even though the conditioned medium reduced the proliferation rate of the MCF-7 cells. Furthermore, high levels of CCL5 and IL- 6 were identified in the conditioned medium of BMSCs pretreated with $40 \mu \mathrm{M}$ rosiglitazone, which may explain the absence of a growth reduction effect on the proliferation rate of MCF-7 cells when the cancer cells were incubated in the conditioned medium. This phenomenon confirmed the negative implications of the use of pioglitazone or rosiglitazone alone, such as the induction of an inflammatory response in treated cells. Notably, this phenomenon was not observed in the BMSCs pretreated with $20 \mu \mathrm{M}$ rosiglitazone $+20 \mu \mathrm{M}$ pioglitazone. The reduced protein levels of FGF4 were only identified in the conditioned medium of BMSCs pretreated with pioglitazone and/or rosiglitazone. Additionally, the pretreatment of BMSCs with $20 \mu \mathrm{M}$ rosiglitazone $+20 \mu \mathrm{M}$ pioglitazone did not increase the levels of CCL5, IL-6 or VEGF in the conditioned medium. These findings indicate that the application of BMSCs pretreated with $20 \mu \mathrm{M}$ pioglitazone $+20 \mu \mathrm{M}$ rosiglitazone to cancer patients via cell-cell interactions may be an effective strategy for the treatment of human breast cancers.

FGF4 is a protein encoded by the FGF4 gene that demonstrates multiple oncogenic activities, including tumor growth and invasion (15). FGF4 is expressed in human breast cancer cells (16). Previous studies demonstrated that the binding site of Oct-4, a POU-domain transcription factor that is markedly expressed in pluripotent embryonic stem cells and exerts an important role in stem cell regulation, was identified in FGF4 (17,18). By studying the expression pattern of FGF4, the present study may lend insights into the regulation of stem cells in carcinogenesis. Consistently with this aim, a reduction in FGF4 was identified in BMSCs pretreated with $20 \mu \mathrm{M}$ pioglitazone $+20 \mu \mathrm{M}$ rosiglitazone, which may have potential therapeutic value for breast cancer, compared with BMSCs 
pretreated with a single TZD. Furthermore, the modification of BMSCs through pretreatment with pioglitazone and rosiglitazone did not change the morphology of the stem cells. This finding indicates that the pretreated stem cells may be injected into the mammary fat pads or bloodstream to reduce cancer cell growth in patients, or may be used in various cell-mediated therapies. Notably, unpublished data in our laboratory revealed that this strategy was more effective when BMSCs were used: A 1.13-fold change in the transcript levels of FGF4 was identified in BMSCs pretreated with pioglitazone and rosiglitazone compared with the control. A decreased effect was demonstrated when the identical treatment was applied to ATSCs (a 1.04-fold change in the FGF4 transcript level). The present study only investigated the expression level of FGF4; other genes that are associated with FGF4, including stromal cell-derived factor 1, platelet-derived growth factor homodimer, epidermal growth factor and basic FGF, will be investigated in future studies.

The approach taken in the present study did not appreciably change the two stem and cancer cell morphologies. Although pretreated stem cells have been shown to have a growth reduction effect on breast cancer cells, there remains a risk that these cells would advance, rather than suppress, cancer. As such, further studies to precisely indicate the potential of pretreated stem cells on breast cancer treatment, including the proliferative and differentiation capacities of the pretreated stem cells, are warranted. To translate these results into a clinical setting, it must be determined how many systemically infused MSCs would actually reach the tumor site, and how long the MSCs would survive in the body post-infusion. Perhaps an in vivo model would be the next optimal approach to prove that this study is clinically relevant.

In conclusion, BMSCs pretreated with pioglitazone and/or rosiglitazone reduced the growth and proliferation rate of breast cancer, an effect that may be attributed to the reduction of the protein level of FGF4 in the pretreated BMSCs. However, further studies on the correlation of the protein levels of FGF4 in the conditioned medium of pioglitazoneand/or rosiglitazone-pretreated BMSCs is warranted.

\section{Acknowledgements}

This project was funded by the Research University Grant Scheme for Individual (RUI) from Universiti Sains Malaysia (no. 1001/CIPPM/811200) for the study of MSCs and the Fundamental Research Grant Scheme (FRGS) Fasa 2/2013 (no. 203/CIPPM/6711336) from the Ministry of HigherEducation (MoHE), Malaysia for the study of FGF4. The pioglitazone study was funded by an Exploratory Research Grant Scheme (ERGS) Fasa 1/2013 (no. 203/CIPPM/6730098). The first author is grateful for the support provided by the Ernst-von-Leyden Scholarship from Berliner Krebsgesellschaft E.V. (to BKG) during her postdoctoral training. The second author thanks the Graduate Assistant Scheme offered by the Institute of Postgraduate Studies (IPS), Universiti Sains Malaysia.

\section{References}

1. Karnoub AE, Dash AB, Vo AP, Sullivan A, Brooks MW, Bell GW, Richardson AL, Polyak K, Tubo R and Weinberg RA: Mesenchymal stem cells within tumour stroma promote breast cancer metastasis. Nature 449: 557-563, 2007.

2. Wang L, Tran I, Seshareddy K, Weiss ML and Detamore MS: A comparison of human bone marrow-derived mesenchymal stem cells and human umbilical cord-derived mesenchymal stromal cells for cartilage tissue engineering. Tissue Eng Part A 15: 2259-2266, 2009.

3. Hombauer H and Minguell JJ: Selective interactions between epithelial tumour cells and bone marrow mesenchymal stem cells. Br J Cancer 82: 1290-1296, 2000.

4. Liu H, Zang C, Fenner MH, Possinger K and Elstner E: PPARgamma ligands and ATRA inhibit the invasion of human breast cancer cells in vitro. Breast Cancer Res Treat 79: 63-74, 2003.

5. Magenta G, Borenstein X, Rolando R and Jasnis MA: Rosiglitazone inhibits metastasis development of a murine mammary tumor cell line LMM3. BMC Cancer 8: 47, 2008.

6. Adams J, Carder PJ, Downey S, Forbes MA, MacLennan K, Allgar V, Kaufman S, Hallam S, Bicknell R, Walker JJ, et al: Vascular endothelial growth factor (VEGF) in breast cancer: Comparison of plasma, serum and tissue VEGF and microvessel density and effects of tamoxifen. Cancer Res 60: 2898-2905, 2000.

7. Robinson SC, Scott KA, Wilson JL, Thompson RG, Proudfoot AE and Balkwill FR: A chemokine receptor antagonist inhibits experimental breast tumor growth. Cancer Res 63: 8360-8365, 2003.

8. Yaal-Hahoshen N, Shina S, Leider-Trejo L, Barnea I, Shabtai EL, Azenshtein E, Greenberg I, Keydar I and Ben-Baruch A: The chemokine CCL5 as a potential prognostic factor predicting disease progression in stage II breast cancer patients. Clin Cancer Res 12: 4474-4480, 2006.

9. Vaday GG, Peehl DM, Kadam PA and Lawrence DM: Expression of CCL5 (RANTES) and CCR5 in prostate cancer. Prostate 66: 124-134, 2006.

10. Loberg RD, Ying C, Craig M, Yan L, Snyder LA and Pienta KJ: CCL2 as an important mediator of prostate cancer growth in vivo through the regulation of macrophage infiltration. Neoplasia 9: 556-562, 2007.

11. Ghosh S, Sullivan CA, Zerkowski MP, Molinaro AM, Rimm DL, Camp RL and Chung GG: High levels of vascular endothelial growth factor and its receptors (VEGFR-1, VEGFR-2, neuropilin-1) are associated with worse outcome in breast cancer. Hum Pathol 39: 1835-1843, 2008.

12. Stacey DL, Gibala MJ, Martin-Ginis KA and Timmons BW: Effects of recovery method after exercise on performance, immune changes and psychological outcomes. J Orthop Sports Phys Ther 40: 656-665, 2010.

13. Hartmann MC, Dwyer RM, Costello M, Potter SM, Curran C, Hennessy E, Newell J, Griffin DG and Kerin MJ: Relationship between CCL5 and transforming growth factor- $\beta 1$ (TGF $\beta 1$ ) in breast cancer. Eur J Cancer 47: 1669-1675, 2011.

14. Liu S, Ginestier C, Ou SJ, Clouthier SG, Patel SH, Monville F, Korkaya H, Heath A, Dutcher J, Kleer CG, et al: Breast cancer stem cells are regulated by mesenchymal stem cells through cytokine networks. Cancer Res 71: 614-624, 2011.

15. Galland F, Stefanova M, Lafage M and Birnbaum D: Localization of the $5^{\prime}$ end of the MCF2 oncogene to human chromosome 15q15-q23. Cytogenet Cell Genet 60: 114-116, 1992.

16. Wang P, Branch DR, Bali M, Schultz GA, Goss PE and Jin T: The POU homeodomain protein OCT3 as a potential transcriptional activator for fibroblast growth factor-4 (FGF-4) in human breast cancer cells. Biochem J 375: 199-205, 2003.

17. Lamb KA and Rizzino A: Effects of differentiation on the transcriptional regulation of the FGF-4 gene: Critical roles played by a distal enhancer. Mol Reprod Dev 51: 218-224, 1998.

18. Boiani $M$ and Schöler HR: Regulatory networks in embryo-derived pluripotent stem cells. Nat Rev Mol Cell Biol 6: 872-884, 2005. 